\section{Cloud Chamber Photographs of Penetrating Showers}

IN a long series of counter experiments, Wataghin, de Souze and Pompia, and Jánossy ${ }^{2}$ and his coworkers have established the existence of showers of penetrating particles different from electron cascade showers and knock-on showers. It now seems probable that the theory of cosmic-ray mesons put forward recently by Hamilton, Heitler and Peng ${ }^{3}$ can account satisfactorily for these showers in terms of mesons (and neutrettos). These particles are assumed to be created by protons (or neutrons), of energies greater than $2 \times 10^{9} \mathrm{eV}$. by cascade or multiple processes.

More direct confirmation of this interpretation of the experimental results is desirable, and for this and Peng if the incident proton (or neutron) has an energy between $2 \times 10^{\circ} \mathrm{eV}$. and $2 \times 10^{10} \mathrm{eV}$. An example of this type of shower is reproduced in Fig. 1.

The second type of shower, two examples of which are reproduced in Fig. 2, consists of penetrating particles coming from different directions. It seems probable that this type of shower is an example of the production of penetrating particles by a cascade process $^{3,4}$.

Bose, Choudhuri and Sinha ${ }^{5}$ have recently published a photograph which they claim shows the production of mesons by a cascade process. To establish such a claim it is first necessary to prove that the shower is not an electron cascade, for electron cascade showers are very frequent while penetrating showers are rare; and it is insufficient to state that the

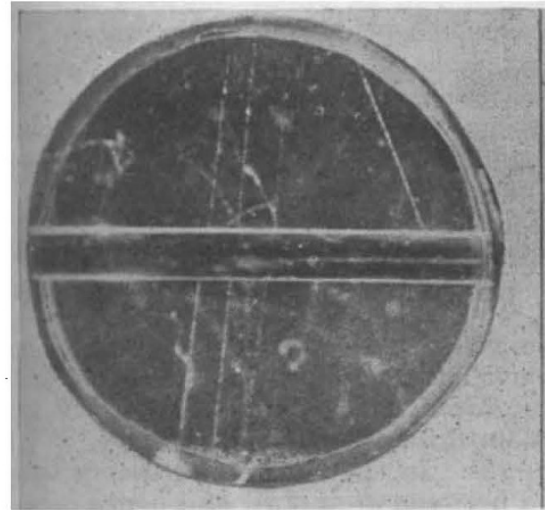

1

FIG. 1. AN EXAMPLE OF A SHOWER OF PENETRATING PARTICles PRODUCED BY A MULTIPIE PROCESS. THREE PARTICLES CAN BE SEEN PASSING THROUGH THE LEAD PLATE.

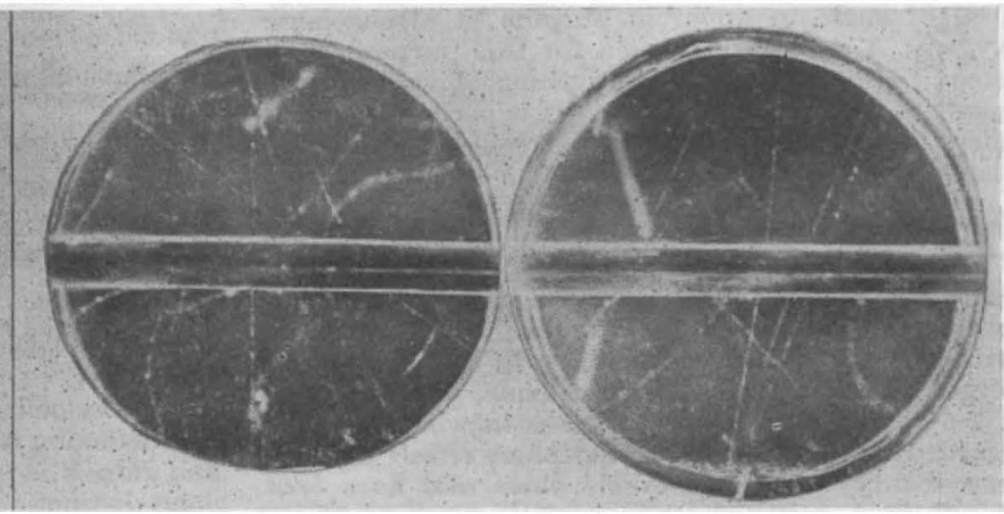

$2 a$

$2 b$

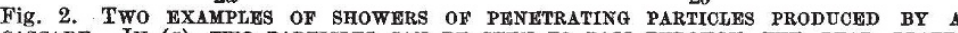
CASOADE. IN (a), TWO PARTICLES CAN BE SREN TO PASS THROUGH THE LEAD PLATE, WHEREAS IN $(b)$ THREE (AND POSSIBLY FOUR) PARTICLES PASS THROUGH THE PLATE. reason we have recently set up a cloud chamber controlled by a set of counters which selects penetrating showers. The counter sets are arranged in three trays and are connected in sevenfold coincidence such that 95 per cent of all coincidences are caused by penetrating showers. The middle and bottom trays are surrounded by lead $15 \mathrm{~cm}$. in thickness, and the total vertical thickness of lead is $53 \mathrm{~cm}$. Electron cascade showers are thus effectively cut out. The top tray is covered with lead $5 \mathrm{~cm}$. in thickness, and the cloud chamber is placed between the middle and bottom trays.

Some thirty photographs have been obtained so far, and of these, eighteen photographs show penetrating particles which pass through a lead block, $2 \cdot 3 \mathrm{~cm}$. in thickness, without multiplication. Nine of the eighteen photographs show showers consisting of two or more penetrating particles.

The results are consistent with the assumption that almost all penetrating showers contain ionizing penetrating particles. Further, they show that the ionizing particles themselves are penetrating, and are not soft ionizing secondaries to a non-ionizing penetrating radiation. The ionizing penetrating particles may, however, be accompanied by non-ionizing particles (for example, neutrettos).

Showers of two main types occur among the nine photographs. The first type, typical of almost all the published photographs of showers of penetrating particles, consists of penetrating particles originating at one point, that is, particles produced by a multiple process. Jánossy ${ }^{2}$ has shown that these showers are to be expected from the theory of Hamilton, Heitler shower contains mesons. Moreover, the one group of particles on Sinha's photograph which clearly passes through the lead plate in the cloud chamber emerges accompanied by several electrons. It is assumed that these are knock-on electrons produced by mesons, but this is very unlikely as the chance of a number of mesons producing several knock-on electrons is negligibly small. The multiplication of the particles in passing through the lead plate is, in fact, a clear indication that they are electrons and not mesons.

Physical Laboratories,

University, Manchester.

G. D. Rochester.

${ }^{1}$ Wataghin, G., de Souze, M., and Pompia, P. A., Phys. Rev., 57, 61, $339(1940)$.

'Jánossy, I., Phys. Rev., 64, 345 (1943).

- Hamilton, J., Heitler, W., and Peng, H. W., Phys. Rev., 64, 78 (1943).

- Peng, H. W., Proc. Roy. Irish Acad., 49 A, 245 (1944).

Bose, D. M., Choudhuri, B., and Sinha, M., Phy8. Rev., 65, 341 (1944).

\section{Volcanic Contributions to the Atmosphere and Ocean}

IF it be assumed, as is now again the fashion, that the nascent earth passed through a liquid stage, it is obvious that "the molten spheroid ... retained, occluded within itself, some large part of the water in the present hydrosphere, as well as much of the carbon dioxide represented by the present carbonates and carbonaceous deposits"1. Most of the carbon dioxide that has become available as a source of 